\title{
Some properties of graded 2-absorbing and graded weakly 2-absorbing submodules
}

\author{
Khaldoun Al-Zoubia,*, Mariam Al-Azaizeh ${ }^{\mathrm{b}}$ \\ ${ }^{a}$ Department of Mathematics and Statistics, Jordan University of Science and Technology, P. O. Box 3030, Irbid 22110, Jordan. \\ ${ }^{b}$ Department of Mathematics, University of Jordan, Amman, Jordan.
}

\begin{abstract}
Let $\mathrm{G}$ be a group with identity $e$. Let $\mathrm{R}$ be a G-graded commutative ring and $M$ a graded R-module. In this paper we will obtain some results concerning the graded 2-absorbing and graded weakly 2-absorbing submodules of a graded modules over a commutative graded ring.
\end{abstract}

Keywords: Graded 2-absorbing submodule, graded weakly 2-absorbing submodule, graded submodules.

2010 MSC: 13A02, 16W50.

(C)2019 All rights reserved.

\section{Introduction and preliminaries}

Throughout this paper all rings are commutative with identity and all modules are unitary.

The concept of weakly prime ideals was initiated by Anderson and Smith in [8]. The concept of weakly 2-absorbing ideals was introduced in [14] as a generalization of the notion of weakly prime ideals. Badawi in [13] introduced the concept of 2-absorbing ideals of commutative rings that is a generalization of the concept of prime ideals. Later on, Anderson and Badawi in [7] generalized the concept of 2-absorbing ideals of commutative rings to the concept of $n$-absorbing ideals of commutative rings for every positive integer $n \geqslant 2$. In light of $[7,13]$ many authors studied the concept of 2-absorbing submodules and $n$ absorbing submodules, (see for example, [15, 18, 24, 26, 27]).

The scope of this paper is devoted to the theory of graded modules over graded commutative rings. One use of rings and modules with gradings is in describing certain topics in algebraic geometry. Graded prime ideals, and graded weakly prime ideals have been studied by various authors, (see for example [5, 10, 25]). The concept of graded 2-absorbing ideals and graded weakly 2-absorbing ideals, generalizations of graded prime ideals, and graded weakly prime ideals, respectively, were studied by Al-Zoubi and Abu-Dawwas, and other authors, (see [2, 19]). Graded prime submodules, and graded weakly prime submodules have been studied by various authors, (see for example $[4,6,9,11,23]$ ). The concept of

\footnotetext{
*Corresponding author

Email addresses: kfzoubi@just.edu.jo (Khaldoun Al-Zoubi), maalazaizeh15@sci.just.edu.jo (Mariam Al-Azaizeh)

doi: $10.22436 /$ jnsa.012.08.01
}

Received: 2018-11-12 Revised: 2019-02-20 Accepted: 2019-02-22 
graded 2-absorbing submodules and graded weakly 2-absorbing submodules, generalizations of graded prime submodules, and graded weakly prime submodules, respectively, were studied by Al-Zoubi and Abu-Dawwas in [1]. Later on, Hamoda and Ashour in [17] introduced the concept of graded n-absorbing submodules that is a generalization of the concept of graded prime ideals.

Here, we study several results concerning of graded 2-absorbing and graded weakly 2-absorbing submodules of graded modules over graded commutative rings.

First, we recall some basic properties of graded rings and modules which will be used in the sequel. We refer to [16, 20-22] for these basic properties and more information on graded rings and modules.

Let $G$ be a group with identity $e$ and $R$ be a commutative ring with identity $1_{R}$. Then $R$ is a G-graded ring if there exist additive subgroups $R_{g}$ of $R$ such that $R=\bigoplus_{g \in G} R_{g}$ and $R_{g} R_{h} \subseteq R_{g h}$ for all $g, h \in G$. The elements of $R_{g}$ are called to be homogeneous of degree $g$ where the $R_{g}$ 's are additive subgroups of $R$ indexed by the elements $g \in G$. If $x \in R$, then $x$ can be written uniquely as $\sum_{g \in G} x_{g}$, where $x_{g}$ is the component of $x$ in $R_{g}$. Moreover, $h(R)=\bigcup_{g \in G} R_{g}$. Let $I$ be an ideal of $R$. Then I is called a graded ideal of $(R, G)$ if $I=\bigoplus_{g \in G}\left(I \cap R_{g}\right)$. Thus, if $x \in I$, then $x=\sum_{g \in G} x_{g}$ with $x_{g} \in I$.

Let $R$ be a $G$-graded ring and $M$ an R-module. We say that $M$ is a G-graded R-module (or graded $R$ module) if there exists a family of subgroups $\left\{M_{g}\right\}_{g \in G}$ of $M$ such that $M=\bigoplus_{g \in G} M_{g}$ (as Abelian groups) and $R_{g} M_{h} \subseteq M_{g h}$ for all $g, h \in G$. Here, $R_{g} M_{h}$ denotes the additive subgroup of $M$ consisting of all finite sums of elements $r_{g} s_{h}$ with $r_{g} \in R_{g}$ and $s_{h} \in M_{h}$. Also, we write $h(M)=\bigcup_{g \in G} M_{g}$ and the elements of $h(M)$ are called to be homogeneous. Let $M=\bigoplus_{g \in G} M_{g}$ be a graded R-module and $N$ a submodule of $M$. Then $N$ is called a graded submodule of $M$ if $N=\bigoplus_{g \in G} N_{g}$, where $N_{g}=N \cap M_{g}$ for $g \in G$. In this case, $N_{g}$ is called the g-component of $N$. Let $R$ be a G-graded ring, $M$ a graded R-module, and $N$ a graded submodule of $M$. Then $(N: R M)$ is defined as $(N: R M)=\{r \in R \mid r M \subseteq N\}$. It is shown in [9, Lemma 2.1] that if $N$ is a graded submodule of $M$, then $(N: R M)=\{r \in R: r M \subseteq N\}$ is a graded ideal of $R$. A proper graded submodule $\mathrm{P}$ of $\mathrm{M}$ is said to be a graded prime submodule (Resp. graded weakly prime submodule) of $\mathrm{M}$ if whenever $r \in h(R)$ and $m \in h(M)$ with $r m \in P($ Resp. $0 \neq r m \in P)$, then either $r \in(P: R M)$ or $m \in P$ (see $[9,11])$. A proper graded ideal I of $\mathrm{R}$ is said to be a graded 2-absorbing ideal (Resp. a graded weakly 2-absorbing ideal) of $R$ if whenever $r, s, t \in h(R)$ with $r s t \in I$ (Resp. $0 \neq r s t \in I$ ), then $r s \in I$ or $r t \in I$ or $s t \in I$ (see [2]). A proper graded submodule $N$ of a graded R-module $M$ is said to be a graded 2-absorbing submodule (Resp. graded weakly 2-absorbing submodule) of $M$ if whenever $r, s \in h(R)$ and $m \in h(M)$ with $r s m \in N($ Resp. $0 \neq r s m \in N)$, then $r s \in(N: R M)$ or $r m \in N$ or $s m \in N$ (see [1]).

\section{Graded 2-absorbing submodules}

Lemma 2.1. Let $\mathrm{R}$ be a $\mathrm{G}$-graded ring, $\mathrm{M}$ a graded $\mathrm{R}$-module, and $\mathrm{N}$ a graded 2-absorbing submodule of $\mathrm{M}$. Let $\mathrm{I}=\bigoplus_{\mathrm{g} \in \mathrm{G}} \mathrm{I}_{\mathrm{g}}$ be a graded ideal of $\mathrm{R}$. Then for every $\mathrm{r} \in \mathrm{h}(\mathrm{R}), \mathrm{m} \in \mathrm{h}(\mathrm{M})$ and $\mathrm{g} \in \mathrm{G}$ with $\mathrm{rI} \mathrm{I}_{\mathrm{g}} \mathrm{m} \subseteq \mathrm{N}$, either $\mathrm{rm} \in \mathrm{N}$ or $\mathrm{I}_{\mathrm{g}} \mathrm{m} \subseteq \mathrm{N}$ or $r \mathrm{I}_{\mathrm{g}} \subseteq\left(\mathrm{N}:_{\mathrm{R}} \mathrm{M}\right)$.

Proof. Let $r \in h(R), m \in h(M)$ and $g \in G$ such that $r I_{g} m \subseteq N, r m \notin N$ and $r I_{g} \not \subseteq(N: R M)$. We have to show that $I_{g} m \subseteq N$. As $r I_{g} \nsubseteq\left(N:_{R} M\right)$, there exists $i_{g} \in I_{g}$ such that $r i_{g} \notin\left(N:_{R} M\right)$. Since $N$ is a graded 2-absorbing submodule, $r i_{g} m \in N, r m \notin N$ and $r i_{g} \notin(N: R M)$, we have $i_{g} m \in N$. Now, let $i_{g}^{\prime} \in I_{g}$. By $i_{g}+i_{g}^{\prime} \in I_{g}$ it follows that $r\left(i_{g}+i_{g}^{\prime}\right) m \in N$. Then either $\left(i_{g}+i_{g}^{\prime}\right) m \in N$ or $r\left(i_{g}+i_{g}^{\prime}\right) \in(N: R M)$ as $N$ is a graded 2-absorbing submodule. If $\left(i_{g}+i_{g}^{\prime}\right) m \in N$, then we get $i_{g}^{\prime} m \in N$ since $i_{g} m \in N$. If $r\left(i_{g}+i_{g}^{\prime}\right) \in\left(N:_{R} M\right)$, then we get $r i_{g}^{\prime} \notin\left(N:_{R} M\right)$ since $r i_{g} \notin\left(N:_{R} M\right)$, but $r i_{g}^{\prime} m \in N$, so $i_{g}^{\prime} m \in N$ since $N$ is a graded 2-absorbing submodule, $r m \notin N$ and $r i_{g}^{\prime} \notin\left(N:_{R} M\right)$. Hence $I_{g} m \subseteq N$.

Theorem 2.2. Let $\mathrm{R}$ be a G-graded ring, $\mathrm{M}$ a graded $\mathrm{R}$-module, and $\mathrm{N}$ a graded 2-absorbing submodule of $\mathrm{M}$. Let $\mathrm{I}=\bigoplus_{\mathrm{g} \in \mathrm{G}} \mathrm{I}_{\mathrm{g}}$ and $\mathrm{J}=\bigoplus_{\mathrm{g} \in \mathrm{G}} \mathrm{J}_{\mathrm{g}}$ be a graded ideals of $\mathrm{R}$. Then for every $\mathrm{m} \in \mathrm{h}(\mathrm{M})$ and $\mathrm{g}, \mathrm{h} \in \mathrm{G}$ with $\mathrm{I}_{\mathrm{g}} \mathrm{J}_{\mathrm{h}} \mathrm{m} \subseteq \mathrm{N}$, either $\mathrm{I}_{\mathrm{g}} \mathrm{m} \subseteq \mathrm{N}$ or $\mathrm{J}_{\mathrm{h}} \mathrm{m} \subseteq \mathrm{N}$ or $\mathrm{I}_{\mathrm{g}} \mathrm{J}_{\mathrm{h}} \subseteq(\mathrm{N}: \mathrm{R} M)$. 
Proof. Let $\mathrm{m} \in \mathrm{h}(\mathrm{M})$ and $\mathrm{g}, \mathrm{h} \in \mathrm{G}$ such that $\mathrm{I}_{\mathrm{g}} \mathrm{J}_{\mathrm{h}} \mathrm{m} \subseteq \mathrm{N}, \mathrm{I}_{\mathrm{g}} \mathrm{m} \nsubseteq \mathrm{N}$ and $\mathrm{J}_{\mathrm{h}} \mathrm{m} \nsubseteq \mathrm{N}$. We have to show that $I_{g} J_{h} \subseteq(N: R M)$. Let $i_{g} \in I_{g}$ and $j_{h} \in J_{h}$. As $I_{g} m \nsubseteq N$ and $J_{h} m \nsubseteq N$, there exist $i_{g}^{\prime} \in I_{g}$ and $j_{h}^{\prime} \in J_{h}$ such that $i_{g}^{\prime} m \notin N$ and $j_{h}^{\prime} m \notin N$. Since $i_{g}^{\prime} J_{h} m \subseteq N, i_{g}^{\prime} m \notin N$ and $J_{h} m \nsubseteq N$, by Lemma 2.1, we get $i_{g}^{\prime} J_{h} \subseteq(N: R M)$. Also since $j_{h}^{\prime} I_{g} m \subseteq N, j_{h}^{\prime} m \notin N$ and $I_{g} m \nsubseteq N$, we get $j_{h}^{\prime} I_{g} \subseteq(N: R M)$, which implies $\left(I_{g} \backslash\left(N:_{R} m\right)\right) J_{h} \subseteq\left(N:_{R} M\right)$ and $\left(J_{h} \backslash\left(N:_{R} m\right)\right) I_{g} \subseteq\left(N:_{R} M\right)$. Hence we have $i_{g}^{\prime} j_{h}^{\prime} \in\left(N:_{R} M\right)$, $i_{g}^{\prime} j_{h} \in(N: R M)$ and $i_{g} j_{h}^{\prime} \in(N: R M)$. By $\left(i_{g}+i_{g}^{\prime}\right) \in I_{g}$ and $\left(j_{h}+j_{h}^{\prime}\right) \in J_{h}$ it follows that $\left(i_{g}+i_{g}^{\prime}\right)\left(j_{h}+\right.$ $\left.j_{h}^{\prime}\right) m \in N$. Since $N$ is a graded 2-absorbing submodule, we get either $\left(i_{g}+i_{g}^{\prime}\right) m \in N$ or $\left(j_{h}+j_{h}^{\prime}\right) m \in N$ or $\left(i_{g}+i_{g}^{\prime}\right)\left(j_{h}+j_{h}^{\prime}\right) \in\left(N:_{R} M\right)$. If $\left(i_{g}+i_{g}^{\prime}\right) m \in N$, then $i_{g} m \notin N$ since $i_{g}^{\prime} m \notin N$, so $i_{g} \in I_{g} \backslash\left(N:_{R} m\right)$. Hence $i_{g} j_{h} \in\left(N:_{R} M\right)$. Similarly, if $\left(j_{h}+j_{h}^{\prime}\right) m \in N$, then $j_{h} m \notin N$ since $j_{h}^{\prime} m \notin N$, therefore $j_{h} \in J_{h} \backslash\left(N:_{R} m\right)$. Hence $i_{g} j_{h} \in\left(N:_{R} M\right)$. If $\left(i_{g}+i_{g}^{\prime}\right)\left(j_{h}+j_{h}^{\prime}\right) \in\left(N:_{R} M\right)$, i.e, $i_{g} j_{h}+i_{g} j_{h}^{\prime}+i_{g}^{\prime} j_{h}+i_{g}^{\prime} j_{h}^{\prime} \in\left(N:_{R} M\right)$, then $i_{g} j_{h} \in(N: R M)$ since $i_{g} j_{h}^{\prime}, i_{g}^{\prime} j_{h}, i_{g}^{\prime} j_{h}^{\prime} \in\left(N:_{R} M\right)$. Thus $I_{g} J_{h} \subseteq\left(N:_{R} M\right)$.

Theorem 2.3. Let $\mathrm{R}$ be a $\mathrm{G}$-graded ring, $\mathrm{M}$ a graded $\mathrm{R}-$ module, and $\mathrm{N}$ be a proper graded submodule of $\mathrm{M}$. Let $\mathrm{I}=\bigoplus_{\mathrm{g} \in \mathrm{G}} \mathrm{I}_{\mathrm{g}}$ and $\mathrm{J}=\bigoplus_{\mathrm{g} \in \mathrm{G}} \mathrm{J}_{\mathrm{g}}$ be graded ideals of $\mathrm{R}$ and $\mathrm{U}=\bigoplus_{\mathrm{g} \in \mathrm{G}} \mathrm{U}_{\mathrm{g}}$ be a graded submodule of $\mathrm{M}$. Then the following statements are equivalent:

(i) $\mathrm{N}$ is a graded 2-absorbing submodule of $\mathrm{M}$;

(ii) for every $\mathrm{g}, \mathrm{h}, \lambda \in \mathrm{G}$ with $\mathrm{I}_{\mathrm{g}} \mathrm{J}_{\mathrm{h}} \mathrm{U}_{\lambda} \subseteq \mathrm{N}$, either $\mathrm{I}_{\mathrm{g}} \mathrm{U}_{\lambda} \subseteq \mathrm{N}$ or $\mathrm{J}_{\mathrm{h}} \mathrm{U}_{\lambda} \subseteq \mathrm{N}$ or $\mathrm{I}_{\mathrm{g}} \mathrm{J}_{\mathrm{h}} \subseteq(\mathrm{N}: \mathrm{R} M)$.

Proof.

(i) $\Rightarrow$ (ii) Assume that $\mathrm{N}$ is a graded 2-absorbing submodule of $M$. Let $g, h, \lambda \in G$ such that $I_{g} J_{h} U_{\lambda} \subseteq N$ and $\mathrm{I}_{g} \mathrm{~J}_{h} \nsubseteq\left(N:_{R} M\right)$. By Theorem 2.2 for all $u_{\lambda} \in \mathrm{U}_{\lambda}$ we have either $\mathrm{I}_{g} \mathrm{u}_{\lambda} \subseteq \mathrm{N}$ or $\mathrm{J}_{h} \mathrm{u}_{\lambda} \subseteq \mathrm{N}$. If $\mathrm{I}_{\mathrm{g}} \mathrm{u}_{\lambda} \subseteq \mathrm{N}$ for all $\mathrm{u}_{\lambda} \in \mathrm{u}_{\lambda}$, then $\mathrm{I}_{\mathrm{g}} \mathrm{u}_{\lambda} \subseteq \mathrm{N}$. Similarly, if $\mathrm{J}_{h} \mathrm{u}_{\lambda} \subseteq \mathrm{N}$ for all $\mathrm{u}_{\lambda} \in \mathrm{u}_{\lambda}$, then $\mathrm{J}_{h} \mathrm{u}_{\lambda} \subseteq \mathrm{N}$. Assume that there exist $u_{\lambda}, u_{\lambda}^{\prime} \in U_{\lambda}$ such that $I_{g} u_{\lambda} \nsubseteq N$ and $J_{h} u_{\lambda}^{\prime} \nsubseteq N$. Since $I_{g} J_{h} u_{\lambda} \subseteq N, I_{g} u_{\lambda} \nsubseteq N$ and $\mathrm{I}_{g} \mathrm{~J}_{h} \nsubseteq(\mathrm{N}: \mathrm{R} M)$, by Theorem 2.2, we get $\mathrm{J}_{h} \mathrm{u}_{\lambda} \subseteq \mathrm{N}$. Also since $\mathrm{I}_{\mathrm{g}} \mathrm{J}_{h} \mathrm{u}_{\lambda}^{\prime} \subseteq \mathrm{N}, \mathrm{J}_{\mathrm{h}} \mathrm{u}_{\lambda}^{\prime} \nsubseteq \mathrm{N}$ and $\mathrm{I}_{\mathrm{g}} \mathrm{J}_{\mathrm{h}} \nsubseteq(\mathrm{N}: \mathrm{R} M)$, we get $\mathrm{I}_{\mathrm{g}} \mathrm{u}_{\lambda}^{\prime} \subseteq \mathrm{N}$. By $\mathrm{u}_{\lambda}+\mathrm{u}_{\lambda}^{\prime} \in \mathrm{U}_{\lambda}$ it follows that $\mathrm{I}_{\mathrm{g}} \mathrm{J}_{\mathrm{h}}\left(\mathrm{u}_{\lambda}+\mathrm{u}_{\lambda}^{\prime}\right) \subseteq \mathrm{N}$. By Theorem 2.2, we get either $\mathrm{I}_{\mathrm{g}}\left(\mathrm{u}_{\lambda}+\mathrm{u}_{\lambda}^{\prime}\right) \subseteq \mathrm{N}$ or $\mathrm{J}_{h}\left(\mathrm{u}_{\lambda}+\mathrm{u}_{\lambda}^{\prime}\right) \subseteq \mathrm{N}$. If $\mathrm{I}_{\mathrm{g}}\left(\mathrm{u}_{\lambda}+\mathrm{u}_{\lambda}^{\prime}\right) \subseteq \mathrm{N}$, then $\mathrm{I}_{\mathrm{g}} \mathrm{u}_{\lambda} \subseteq \mathrm{N}$ since $\mathrm{I}_{\mathrm{g}} \mathrm{u}_{\lambda}^{\prime} \subseteq \mathrm{N}$ which is a contradiction. Similarly if $J_{h}\left(u_{\lambda}+u_{\lambda}^{\prime}\right) \subseteq N$, then $J_{h} u_{\lambda}^{\prime} \subseteq N$ since $J_{h} u_{\lambda} \subseteq N$, a contradiction. Therefore either $\mathrm{I}_{\mathrm{g}} \mathrm{U}_{\lambda} \subseteq \mathrm{N}$ or $\mathrm{J}_{\mathrm{h}} \mathrm{U}_{\lambda} \subseteq \mathrm{N}$.

(ii) $\Rightarrow$ (i) Assume that (ii) holds. Let $r_{g}, s_{h} \in h(R)$ and $m_{\lambda} \in h(M)$ such that $r_{g} s_{h} m_{\lambda} \in N$. Let I $=r_{g} R$ and $J=s_{h} R$ be graded ideals of $R$ generated by $r_{g}, s_{h}$, respectively and $U_{\lambda}=m_{\lambda} R$ be a graded submodule of $M$ generated by $m_{\lambda}$. Then $I_{g} J_{h} U_{\lambda} \subseteq N$. By our assumption we obtain $I_{g} U_{\lambda} \subseteq N$ or $J_{h} U_{\lambda} \subseteq N$ or $\mathrm{I}_{\mathrm{g}} \mathrm{J}_{\mathrm{h}} \subseteq(\mathrm{N}: \mathrm{R} M)$. Hence $r_{\mathrm{g}} \mathrm{m}_{\lambda} \in \mathrm{N}$ or $\mathrm{s}_{\mathrm{h}} \mathrm{m}_{\lambda} \in N$ or $\mathrm{r}_{\mathrm{g}} \mathrm{s}_{\mathrm{h}} \in\left(\mathrm{N}:_{\mathrm{R}} M\right)$. Therefore $\mathrm{N}$ is a graded 2-absorbing submodule of $M$.

\section{Graded weakly 2-absorbing submodules}

Let $N$ be a graded submodule of $M$ and let $g \in G$. We say that $N_{g}$ is a weakly g-2-absorbing submodule of $R_{e}$-module $M_{g}$, if $N_{g} \neq M_{g}$; and whenever $r, s \in R_{e}$ and $m \in M_{g}$ with $0 \neq r s m \in N_{g}$, then either $r s \in\left(N_{g}: R_{e} M_{g}\right)$ or $r m \in N_{g}$ or $s m \in N_{g}$ (see [1]).

Lemma 3.1. Let $\mathrm{R}$ be a $\mathrm{G}$-graded ring, $\mathrm{M}$ a graded $\mathrm{R}$-module and $\mathrm{N}$ a graded weakly 2-absorbing submodule of $\mathrm{M}$, and $\mathrm{g} \in \mathrm{G}$. If $\mathrm{r}_{\mathrm{e}} \mathrm{s}_{\mathrm{e}} \mathrm{U} \subseteq \mathrm{N}_{\mathrm{g}}$ and $0 \neq 2 \mathrm{r}_{\mathrm{e}} \mathrm{s}_{\mathrm{e}} \mathrm{U}$ for some $\mathrm{r}_{e}, \mathrm{~s}_{\mathrm{e}} \in \mathrm{R}_{\mathrm{e}}$ and some submodule $\mathrm{U}$ of $\mathrm{M}_{\mathrm{g}}$, then either $\mathrm{r}_{\mathrm{e}} \mathrm{s}_{\mathrm{e}} \in\left(\mathrm{N}_{\mathrm{g}}:_{\mathrm{R}_{e}} \mathrm{M}_{\mathrm{g}}\right)$ or $\mathrm{r}_{\mathrm{e}} \mathrm{U} \subseteq \mathrm{N}_{\mathrm{g}}$ or $\mathrm{s}_{\mathrm{e}} \mathrm{U} \subseteq \mathrm{N}_{\mathrm{g}}$.

Proof. By [1, Lemma 3.2], $N_{g}$ is a weakly g-2-absorbing $R_{e}$-submodule of $M_{g}$ for every $g \in G$. Assume that $r_{e} s_{e} U \subseteq N_{g}, 0 \neq 2 r_{e} s_{e} U$ and $r_{e} s_{e} \notin\left(N_{g}: R_{e} M_{g}\right)$ for some $r_{e}, s_{e} \in R_{e}$ and some submodule $U$ of $M_{g}$. We have to show that $U \subseteq\left(N_{g}: M_{g} r_{e}\right) \cup\left(N_{g}: M_{g} s_{e}\right)$. Let $u_{g} \in U \subseteq M_{g}$. If $0 \neq r_{e} s_{e} u_{g}$, then either $r_{e} u_{g} \in N_{g}$ or $s_{e} u_{g} \in N_{g}$ since $N_{g}$ is a weakly g-2-absorbing $R_{e}$-submodule of $M_{g}$ and $r_{e} s_{e} \notin\left(N_{g}: R_{e} M_{g}\right)$. So $u_{g} \in\left(N_{g}: M_{g} r_{e}\right) \cup\left(N_{g}:_{M_{g}} s_{e}\right)$. Suppose that $r_{e} s_{e} u_{g}=0$. Since $0 \neq 2 r_{e} s_{e} u$, there exists $u_{g}^{\prime} \in U \subseteq M_{g}$ such that $0 \neq 2 r_{e} s_{e} u_{g}^{\prime}$, hence $0 \neq r_{e} s_{e} u_{g}^{\prime} \in N_{g}$. Since $N_{g}$ is a weakly g-2-absorbing $R_{e}$-submodule of $M_{g}$, 
we have either $r_{e} u_{g}^{\prime} \in N_{g}$ or $s_{e} u_{g}^{\prime} \in N_{g}$. Let $v_{g}=u_{g}+u_{g}^{\prime}$. Hence $0 \neq r_{e} s_{e} v_{g} \in N_{g}$. Then $r_{e} v_{g} \in N_{g}$ or $s_{e} v_{g} \in N_{g}$ as $N_{g}$ is a weakly g-2-absorbing $R_{e}$-submodule of $M_{g}$. Now, we consider three cases.

Case 1: $r_{e} u_{g}^{\prime} \in N_{g}$ and $s_{e} u_{g}^{\prime} \notin N_{g}$. On the contrary let $r_{e} u_{g} \notin N_{g}$. Then $r_{e} v_{g} \notin N_{g}$ and hence $s_{e} v_{g} \in N_{g}$. This yields that $r_{e}\left(v_{g}+u_{g}^{\prime}\right) \notin N_{g}$ and $s_{e}\left(v_{g}+u_{g}^{\prime}\right) \notin N_{g}$. So $0=r_{e} s_{e}\left(v_{g}+u_{g}^{\prime}\right)=2 r_{e} s_{e} u_{g}^{\prime}$ since $N_{g}$ is a weakly $g$-2-absorbing submodule and $r_{e} s_{e} \notin\left(N_{g}: R_{e} M_{g}\right)$, which is a contradiction. Thus $r_{e} u_{g} \in N_{g}$.

Case 2: $r_{e} u_{g}^{\prime} \notin N_{g}$ and $s_{e} u_{g}^{\prime} \in N_{g}$. The proof is similar to that of Case 1.

Case 3: $r_{e} u_{g}^{\prime} \in N_{g}$ and $s_{e} u_{g}^{\prime} \in N_{g}$. Since $r_{e} v_{g} \in N_{g}$ or $s_{e} v_{g} \in N_{g}$, we get $r_{e} u_{g} \in N_{g}$ or $s_{e} u_{g} \in N_{g}$. Thus $u_{g} \in\left(N_{g}: M_{g} r_{e}\right) \cup\left(N_{g}:_{M_{g}} s_{e}\right)$.

Theorem 3.2. Let $\mathrm{R}$ be a $\mathrm{G}$-graded ring, $\mathrm{M}$ a graded $\mathrm{R}$-module, and $\mathrm{N}$ a graded weakly 2-absorbing submodule of $\mathrm{M}$ and $\mathrm{g} \in \mathrm{G}$. If $\mathrm{r}_{e} \mathrm{IU} \subseteq \mathrm{N}_{\mathrm{g}}$ and $0 \neq 4 \mathrm{r}_{e} \mathrm{IU}$ for some $\mathrm{r}_{e} \in \mathrm{R}_{e}$, I ideal of $\mathrm{R}_{e}$ and some submodule $\mathrm{U}$ of $\mathrm{M}_{\mathrm{g}}$, then either $\mathrm{r}_{e} \mathrm{I} \subseteq\left(\mathrm{N}_{\mathrm{g}}: \mathrm{R}_{e} \mathrm{M}_{\mathrm{g}}\right)$ or $\mathrm{r}_{\mathrm{e}} \mathrm{U} \subseteq \mathrm{N}_{\mathrm{g}}$ or $\mathrm{IU} \subseteq \mathrm{N}_{\mathrm{g}}$.

Proof. By [1, Lemma 3.2], $N_{g}$ is a weakly g-2-absorbing $R_{e}$-submodule of $M_{g}$ for every $g \in G$. Assume that $r_{e} I U \subseteq N_{g}, 0 \neq 4 r_{e} I U, r_{e} I \nsubseteq\left(N_{g}: R_{e} M_{g}\right)$ and $r_{e} U \nsubseteq N_{g}$ for some $r_{e} \in R_{e}$, I ideal of $R_{e}$ and some submodule $\mathrm{U}$ of $\mathrm{M}_{\mathrm{g}}$. We have to show that IU $\subseteq \mathrm{N}_{\mathrm{g}}$. By [3, Lemma 2.15], there exists $s_{e} \in \mathrm{I}$ such that $0 \neq 2 r_{e} s_{e} U+2 r_{e} s_{e} U$ and $r_{e} s_{e} \notin\left(N_{g}: R_{e} M_{g}\right)$. Hence $0 \neq 2 r_{e} s_{e} U$ and $r_{e} s_{e} U \subseteq N_{g}$. Thus $s_{e} U \subseteq N_{g}$ by Lemma 3.1. Let $i_{e} \in I$. Assume that $0 \neq 2 r_{e} i_{e} U$. Since $r_{e} i_{e} U \subseteq N_{g}$ and $r_{e} U \nsubseteq N_{g}$, by Lemma 3.1, we have either $r_{e} i_{e} \in\left(N_{g}: R_{e} M_{g}\right)$ or $i_{e} U \subseteq N_{g}$. Thus $i_{e} \in\left(\left(N_{g}: R_{e} M_{g}\right): R_{e} r_{e}\right) \cup\left(N_{g}: R_{e} U\right)$. Now, let $2 r_{e} i_{e} U=0$. This yields that $0 \neq 2 r_{e} s_{e} U=2 r_{e}\left(s_{e}+i_{e}\right) U$ and $r_{e}\left(s_{e}+i_{e}\right) U \subseteq N_{g}$. It follows that either $\left(s_{e}+i_{e}\right) \mathrm{U} \subseteq \mathrm{N}_{\mathrm{g}}$ or $\mathrm{r}_{e}\left(s_{e}+i_{e}\right) \in\left(\mathrm{N}_{\mathrm{g}}: \mathrm{R}_{e} \mathrm{M}_{\mathrm{g}}\right)$ by Lemma 3.1. If $\left(s_{e}+i_{e}\right) \mathrm{U} \subseteq \mathrm{N}_{\mathrm{g}}$, then since $s_{e} \mathrm{U} \subseteq \mathrm{N}_{\mathrm{g}}$, we get $i_{e} U \subseteq N_{g}$. Let $r_{e}\left(s_{e}+i_{e}\right) \in\left(N_{g}: R_{e} M_{g}\right)$ and $\left(s_{e}+i_{e}\right) U \nsubseteq N_{g}$. Then $\left(s_{e}+i_{e}+s_{e}\right) U \nsubseteq N_{g}$ since $s_{e} U \subseteq N_{g}$. As $r_{e} s_{e} \notin\left(N_{g}: R_{e} M_{g}\right)$ and $r_{e}\left(s_{e}+i_{e}\right) \in\left(N_{g}: R_{e} M_{g}\right)$, then $r_{e}\left(s_{e}+i_{e}+s_{e}\right) \notin\left(N_{g}: R_{e} M_{g}\right)$. Since $2 r_{e}\left(s_{e}+i_{e}+s_{e}\right) U=4 r_{e} s_{e} U \neq 0$ and $r_{e}\left(s_{e}+i_{e}+s_{e}\right) U \subseteq N_{g}$, by Lemma 3.1, we get $r_{e} U \subseteq N_{g}$, which is a contradiction. Hence $\left(s_{e}+i_{e}\right) U \subseteq N_{g}$ and so $i_{e} U \subseteq N_{g}$. Thus $I \subseteq\left(\left(N_{g}: R_{e} M_{g}\right): R_{e} r_{e}\right) \cup\left(N_{g}: R_{e} U\right)$. This yields that $\mathrm{IU} \subseteq \mathrm{N}_{\mathrm{g}}$ since $\mathrm{r}_{e} \mathrm{I} \nsubseteq\left(\mathrm{N}_{\mathrm{g}}: \mathrm{R}_{e} M_{g}\right)$.

Let $R_{i}$ be a graded commutative ring with identity and $M_{i}$ be a graded $R_{i}$-module for $i=1,2$. Let $R=R_{1} \times R_{2}$. Then $M=M_{1} \times M_{2}$ is a graded R-module and each graded submodule of $M$ is of the form $N=N_{1} \times N_{2}$ for some graded submodules $N_{1}$ of $M_{1}$ and $N_{2}$ of $M_{2}$.

Theorem 3.3. Let $\mathrm{R}=\mathrm{R}_{1} \times \mathrm{R}_{2}$ be a G-graded ring and $\mathrm{M}=\mathrm{M}_{1} \times \mathrm{M}_{2}$ be a graded R-module where $\mathrm{M}_{1}$ is a graded $R_{1}$-module and $M_{2}$ is a graded $R_{2}$-module. Let $N_{1}$ be a proper graded submodule of $M_{1}$. Then the following statements are equivalent:

(i) $\mathrm{N}_{1}$ is a graded 2-absorbing of $\mathrm{M}_{1}$;

(ii) $\mathrm{N}_{1} \times \mathrm{M}_{2}$ is a graded 2-absorbing submodule of $\mathrm{M}$;

(iii) $\mathrm{N}_{1} \times \mathrm{M}_{2}$ is a graded weakly 2-absorbing submodule of $\mathrm{M}$.

Proof.

(i) $\Rightarrow$ (ii) Assume that $\left(r_{1}, r_{2}\right)\left(s_{1}, s_{2}\right)\left(m_{1}, m_{2}\right)=\left(r_{1} s_{1} m_{1}, r_{2} s_{2} m_{2}\right) \in N_{1} \times M_{2}$, where $r_{1}, s_{1} \in h\left(R_{1}\right), r_{2}, s_{2} \in$ $h\left(R_{2}\right), m_{1} \in h\left(M_{1}\right), m_{2} \in h\left(M_{2}\right)$. Then $r_{1} s_{1} m_{1} \in N_{1}$. Since $N_{1}$ is a graded 2-absorbing of $M_{1}$, we get either $r_{1} m_{1} \in N_{1}$ or $s_{1} m_{1} \in N_{1}$ or $r_{1} s_{1} \in\left(N_{1}: R_{1} M_{1}\right)$. If $r_{1} m_{1} \in N_{1}$, then $\left(r_{1}, r_{2}\right)\left(m_{1}, m_{2}\right)=\left(r_{1} m_{1}, r_{2} m_{2}\right) \in N_{1} \times$ $M_{2}$. Similarly, if $s_{1} m_{1} \in N_{1}$, then $\left(s_{1}, s_{2}\right)\left(m_{1}, m_{2}\right)=\left(s_{1} m_{1}, s_{2} m_{2}\right) \in N_{1} \times M_{2}$. Again, if $r_{1} s_{1} \in\left(N_{1}: R_{1} M_{1}\right)$, then $\left(r_{1}, r_{2}\right)\left(s_{1}, s_{2}\right)=\left(r_{1} s_{1}, r_{2} s_{2}\right) \in\left(N_{1} \times M_{2}: R M\right)$. Thus $N_{1} \times M_{2}$ is a graded 2-absorbing submodule of M.

(ii) $\Rightarrow$ (iii) It is obvious.

(iii) $\Rightarrow$ (i) Let $r_{1} s_{1} m_{1} \in N_{1}$ for $r_{1}, s_{1} \in h\left(R_{1}\right)$ and $m_{1} \in h\left(M_{1}\right)$. Then for each $0 \neq m_{2} \in h\left(M_{2}\right)$, we have $(0,0) \neq\left(r_{1}, 1\right)\left(s_{1}, 1\right)\left(m_{1}, m_{2}\right)=\left(r_{1} s_{1} m_{1}, m_{2}\right) \in N_{1} \times M_{2}$. Since $N_{1} \times M_{2}$ is a graded weakly 2-absorbing submodule of $M$, we get either $\left(r_{1}, 1\right)\left(m_{1}, m_{2}\right)=\left(r_{1} m_{1}, m_{2}\right) \in N_{1} \times M_{2}$ or $\left(s_{1}, 1\right)\left(m_{1}, m_{2}\right)=\left(s_{1} m_{1}, m_{2}\right) \in$ $N_{1} \times M_{2}$ or $\left(r_{1}, 1\right)\left(s_{1}, 1\right)=\left(r_{1} s_{1}, 1\right) \in\left(N_{1} \times M_{2}: R M\right)$. It follows that either $r_{1} m_{1} \in N_{1}$ or $s_{1} m_{1} \in N_{1}$ or $r_{1} s_{1} \in\left(N_{1}: R_{1} M_{1}\right)$. 
Theorem 3.4. Let $R=R_{1} \times R_{2}$ be a $\mathrm{G}$-graded ring and $M=M_{1} \times M_{2}$ be a graded $R$-module where $M_{1}$ is a nonzero graded $R_{1}$-module and $\mathrm{M}_{2}$ is a nonzero graded $\mathrm{R}_{2}$-module. Let $\mathrm{N}_{1}$ and $\mathrm{N}_{2}$ be proper graded submodules of $\mathrm{M}_{1}$ and $\mathrm{M}_{2}$, respectively.

(i) If $\mathrm{N}=\mathrm{N}_{1} \times \mathrm{N}_{2}$ is a graded weakly 2-absorbing submodule of $\mathrm{M}$, then $\mathrm{N}_{1}$ is a graded weakly prime submodule of $\mathrm{M}_{1}$; moreover, if $0 \neq \mathrm{N}_{2}$, then $\mathrm{N}_{1}$ is a graded classical prime submodule of $\mathrm{M}_{1}$.

(ii) If $\mathrm{N}=\mathrm{N}_{1} \times \mathrm{N}_{2}$ is a graded weakly 2-absorbing submodule of $\mathrm{M}$ and $\left(\mathrm{N}_{1}: \mathrm{R}_{1} \mathrm{M}_{1}\right) \mathrm{M}_{1} \neq 0$, then $\mathrm{N}_{2}$ is a graded prime submodule of $\mathrm{M}_{2}$.

Proof.

(i) Assume that $\mathrm{N}=\mathrm{N}_{1} \times \mathrm{N}_{2}$ is a graded weakly 2-absorbing submodule of $M$. We show that $N_{1}$ is a graded weakly prime submodule of $M_{1}$. Since $N_{2} \neq M_{2}$, there exists $m_{2} \in h\left(M_{2}\right) \backslash N_{2}$. Let $0 \neq r m_{1} \in N_{1}$ for $r \in h\left(R_{1}\right)$ and $m_{1} \in h\left(M_{1}\right)$. Then $(0,0) \neq(r, 1)(1,0)\left(m_{1}, m_{2}\right)=\left(r m_{1}, 0\right) \in N=N_{1} \times N_{2}$. Since $N=N_{1} \times N_{2}$ is a graded weakly 2-absorbing submodule of $M$ and $m_{2} \notin N_{2}$, either $(r, 1)(1,0)=(r, 0) \in$ $(N: R \quad M)=\left(N_{1}: R_{1} M_{1}\right) \times\left(N_{2}: R_{2} M_{2}\right)$ or $(1,0)\left(m_{1}, m_{2}\right)=\left(m_{1}, 0\right) \in N=N_{1} \times N_{2}$. Hence either $m_{1} \in N_{1}$ or $r \in\left(N_{1}: R M_{1}\right)$ which shows that $N_{1}$ is a graded weakly prime submodule of $M_{1}$. Now assume that $0 \neq N_{2}$ and let $r s m_{1} \in N_{1}$ for $r, s \in h\left(R_{1}\right)$ and $m_{1} \in h\left(M_{1}\right)$. Let $0 \neq n_{2} \in N_{2} \cap h\left(M_{2}\right)$. Then $(0,0) \neq(r, 1)(s, 1)\left(m_{1}, n_{2}\right)=\left(r s m_{1}, n_{2}\right) \in N=N_{1} \times N_{2}$. Since $N=N_{1} \times N_{2}$ is a graded weakly 2-absorbing submodule of $M$ and $1 \notin\left(N_{2}: R M_{2}\right)$, we get either $(r, 1)\left(m_{1}, n_{2}\right)=\left(r m_{1}, n_{2}\right) \in N=N_{1} \times N_{2}$ or $(s, 1)\left(m_{1}, n_{2}\right)=\left(s m_{1}, n_{2}\right) \in N=N_{1} \times N_{2}$. Hence, either $r m_{1} \in N_{1}$ or $s m_{1} \in N_{1}$. Therefore $N_{1}$ is a graded classical prime submodule of $M_{1}$.

(ii) Assume that $N=N_{1} \times N_{2}$ is a graded weakly 2-absorbing submodule of $M$ and $\left(N_{1}: R_{1} M_{1}\right) M_{1} \neq 0$. Let $r m_{2} \in N_{2}$ for $r \in h\left(R_{2}\right)$ and $m_{2} \in h\left(M_{2}\right)$. If $\left(N_{1}: R_{1} \quad M_{1}\right) m_{1}=0$ for each $m_{1} \in M_{1} \backslash N_{1}$, then $\left(M_{1} \backslash N_{1}\right) \subseteq\left(0: M_{1}\left(N_{1}: R_{1} M_{1}\right)\right)$. Thus $M_{1}=N_{1} \cup\left(M_{1} \backslash N_{1}\right) \subseteq N_{1} \cup\left(0: M_{1}\left(N_{1}: R_{1} M_{1}\right)\right)$ and since $M_{1} \nsubseteq N_{1}$, we get $M_{1} \subseteq\left(0: M\left(N_{1}: R_{1} M_{1}\right)\right)$ by [12, Lemma 2.2]. Hence $\left(N_{1}: R_{1} M_{1}\right) M_{1}=0$, which is a contradiction. Thus there exist $t \in\left(N_{1}: R_{1} M_{1}\right) \cap h\left(R_{1}\right)$ and $m_{1} \in h\left(M_{1}\right) \backslash N_{1}$ with $t m_{1} \neq 0$. Then $(0,0) \neq$ $(t, 1)(1, r)\left(m_{1}, m_{2}\right)=\left(t m_{1}, r m_{2}\right) \in N=N_{1} \times N_{2}$. Since $N=N_{1} \times N_{2}$ is a graded weakly 2-absorbing submodule of $M$ and $m_{1} \notin N_{1}$, we get $(t, 1)(1, r)=(t, r) \in(N: R M)=\left(N_{1}: R_{1} M\right) \times\left(N_{2}: R_{2} M_{2}\right)$ or $(t, 1)\left(m_{1}, m_{2}\right)=\left(t m_{1}, m_{2}\right) \in N$. It follows that either $r \in\left(N_{2}: R_{2}, M_{2}\right)$ or $m_{2} \in N_{2}$. Therefore $N_{2}$ is a graded prime submodule of $\mathrm{M}_{2}$.

Theorem 3.5. Let $\mathrm{R}=\mathrm{R}_{1} \times \mathrm{R}_{2}$ be a G-graded ring and $\mathrm{M}=\mathrm{M}_{1} \times \mathrm{M}_{2}$ be a graded R-module where $\mathrm{M}_{1}$ is a nonzero graded $R_{1}$-module and $M_{2}$ is a nonzero graded $R_{2}$-module. Let $0 \neq N_{1}$ be a proper graded submodule of $M_{1}$ and $\left(N_{1}: R_{1} M_{1}\right) M_{1} \neq 0$. Then the graded submodule $N_{1} \times 0$ is a graded weakly 2-absorbing submodule of $M_{1}$ if and only if $\mathrm{N}_{1}$ is a graded weakly prime submodule of $\mathrm{M}_{1}$ and 0 is a graded prime submodule of $\mathrm{M}_{2}$.

Proof.

$(\Rightarrow)$ By Theorem 3.4.

$(\Leftarrow)$ Assume that $(0,0) \neq\left(r_{1}, r_{2}\right)\left(s_{1}, s_{2}\right)\left(m_{1}, m_{2}\right)=\left(r_{1} s_{1} m_{1}, r_{2} s_{2} m_{2}\right) \in N_{1} \times 0$, where $r_{1}, s_{1} \in h\left(R_{1}\right), r_{2}, s_{2} \in$ $h\left(R_{2}\right), m_{1} \in h\left(M_{1}\right), m_{2} \in h\left(M_{2}\right)$. Then $0 \neq r_{1} s_{1} m_{1} \in N_{1}$ and $r_{2} s_{2} m_{2}=0$. Since $N_{1}$ is a graded weakly prime submodule of $M_{1}$, we get either $r_{1} \in\left(N_{1}: R_{1} M_{1}\right)$ or $s_{1} \in\left(N_{1}: R_{1} M_{1}\right)$ or $m_{1} \in N_{1}$. Since 0 is a graded prime submodule of $M_{2}$ and $r_{2} s_{2} m_{2}=0$, we get either $r_{2} \in\left(0: R_{2} M_{2}\right)$ or $s_{2} \in\left(0: R_{2} M_{2}\right)$ or $m_{2}=0$. It is easy to see that in any of the above cases $\left(r_{1}, r_{2}\right)\left(s_{1}, s_{2}\right) \in\left(N_{1} \times 0: R \quad M\right)$ or $\left(r_{1}, r_{2}\right)\left(m_{1}, m_{2}\right) \in N_{1} \times 0$ or $\left(s_{1}, s_{2}\right)\left(m_{1}, m_{2}\right) \in N_{1} \times 0$. Thus $N_{1} \times 0$ is a graded weakly 2-absorbing submodule of $M_{1}$.

\section{Acknowledgment}

The authors wish to thank sincerely the referees for their valuable comments and suggestions. 


\section{References}

[1] K. Al-Zoubi, R. Abu-Dawwas, On graded 2-absorbing and weakly graded 2-absorbing submodules, J. Math. Sci. Adv. Appl., 28 (2014), 45-60. 1, 3, 3, 3

[2] K. Al-Zoubi, R. Abu-Dawwas, S. Ceken, On graded 2-absorbing and graded weakly 2-absorbing ideals, Hacet. J. Math. Stat., 2018 (2018), 11 pages. 1

[3] K. Al-Zoubi, M. Al-Azaizeh, On graded weakly 2-absorbing primary submodules, Vietnam J. Math., 2018 (2018), 1-11. 3

[4] K. Al-Zoubi, M. Jaradat, R. Abu-Dawwa, On graded classical prime and graded prime submodules, Bull. Iranian Math. Soc., 41 (2015), 217-225. 1

[5] K. Al-Zoubi, F. Qarqaz, An intersection condition for graded prime ideals, Boll. Unione Mat. Ital., 11 (2018), 483-488. 1

[6] K. Al-Zoubi, B. Rabab'a, Some properties of Graded prime and graded weakly prime submodules, Far East J. Math. Sci. (FJMS), 102 (2017), 1571-1829. 1

[7] D. F. Anderson, A. Badawi, On n-absorbing ideals of commutative rings, Comm. Algebra, 39 (2011), 1646-1672. 1

[8] D. D. Anderson, E. Smith, Weakly prime ideals, Houston J. Math., 29 (2003), 831-840. 1

[9] S. E. Atani, On graded prime submodules, Chiang Mai. J. Sci., 33 (2006), 3-7. 1

[10] S. E. Atani, On graded weakly prime ideals, Turkish J. Math., 30 (2006), 351-358. 1

[11] S. E. Atani, On graded weakly prime submodules, Int. Math. Forum, 1 (2006), 61-66. 1

[12] S. E. Atani, U. Tekir, On the graded primary avoidance theorem, Chiang Mai J. Sci., 34 (2007), 161-164. 3

[13] A. Badawi, On 2-absorbing ideals of commutative rings, Bull. Austral. Math. Soc., 75 (2007), 417-429. 1

[14] A. Badawi, A. Yousefian Darani, On weakly 2-absorbing ideals of commutative rings, Houston J. Math., 39 (2013), 441-452. 1

[15] M. K. Dubey, P. Aggarwal, On 2-absorbing submodules over commutative rings, Lobachevskii J. Math., 36 (2015), 58-64. 1

[16] R. Hazrat, Graded Rings and Graded Grothendieck Groups, Cambridge University Press, Cambridge, (2016). 1

[17] H. Mohammad, A. E. Ashour, On graded n-absorbing submodules, Matematiche (Catania), 70 (2015), 243-254. 1

[18] S. Moradi, A. Azizi, Weakly 2-absorbing submodules of modules, Turkish J. Math., 40 (2016), 350-364. 1

[19] S. R. Naghani, H. F. Moghimi, On Graded 2-Absorbing and Graded Weakly 2-Absorbing Ideals of a Commutative Ring, Çankaya Üniversitesi Bilim ve Mühendislik Dergisi, 13 (2016), 11-17. 1

[20] C. Nǎstǎsescu, F. Van Oystaeyen, Graded and filtered rings and modules, Springer, Berlin, (1979). 1

[21] C. Nǎstǎsescu, F. Van Oystaeyen, Graded Ring Theory, North-Holland Publishing Co., Amsterdam-New York, (1982).

[22] C. Nǎstǎsescu, F. Van Oystaeyen, Methods of Graded Rings, Springer-Verlag, Berlin, (2004). 1

[23] K. H. Oral, Ü. Tekir, A. G. Ağargün, On graded prime and primary submodules, Turkish J. Math., 35 (2011), $159-167$. 1

[24] S. Payrovi, S. Babaei, On the 2-absorbing submodules, Iran. J. Math. Sci. Inform., 10 (2015), 131-137. 1

[25] M. Refai, K. Al-Zoubi, On graded primary ideals, Turkish J. Math., 28 (2004), 217-230. 1

[26] A. Yousefian Darani, F. Soheilnia, On 2-absorbing and weakly 2-absorbing submodules, Thai J. Math., 9 (2012), $577-584$. 1

[27] A. Yousefian Darani, F. Soheilnia, On n-absorbing submodules, Math. Commun., 17 (2012), 547-557. 1 